\section{NUCLEIC ACIDS AND PROTEINS}

\section{Genetic Elements}

Properties and Functions. Edited by D. Shugar. (Sym. posium held on the occasion of the Third Meeting of the Federation of European Biochemical Societies, Warsaw, April 4-7, 1966.) Pp. ix +361 . (London: Academic Press, Inc. (London), Ltd.; New York: Academic Press, Inc.; Warsaw: PWN-Polish Scientific Publishers, 1967.) 84s.; \$15.

THE book is a fascinating collection of fifteen articles concerned with recent developments in the structure of nucleic acids, their replication, the genetic code and the mechanism of protein synthesis. The organizers of the symposium attempted to select topics which were of direct interest not only to the specialists but also to as many of the participating biochemists as could be envis. aged. It is perhaps partly because of this policy that, despite the title, there is little concern with chromosomes, mutation, recombination, genetic suppression, regulatory mechanisms or the transeription of DNA into RNA. Nevertheless, the limited range has enabled the editor and contributors to produce a valuable and coherent book.

Several papers are concerned with the structure of nucleic acids. Fuller reviews the conclusions obtained from X-ray work on solid materials and includes an interesting study of the interaction between DNA and certain antibiotics. Luzzati, Witz and Mathis describe the value of $\mathrm{X}$-ray techniques in determining the conformation of nucleic acids in solution. G. L. Brown, Lee and Metz discuss the study of active sites in RNA by chemical modification. Zachau, Dütting and Feldmann give an account of the techniques used in their determination of the primary sequence of serine transfer RNA and undertaken simultaneously with Holley's work on alanine RNA. Engelhardt and his collaborators describe important parallel Russian work on the valine transfer RNA leading to a probable primary structure which is essentially confirmed by the results of further work added at the proof stage. Perhaps because of a shortage of time at the meeting, neither of these two papers refers to conceivable secondary structures of transfer RNA such as the cloverleaf model that is now receiving much attention. In an important short paper, Sanger and Brownlee describe their extremely powerful methods for the fractionation and identification of radioactive oligonucleotides. These methods have more recently (Nature, 215, 735; 1967) led to the determination of the primary sequence of the $5 S$ ribosomal RNA.

Borst, Kroon and Ruttenberg describe their own and other work on mitochondrial DNA and consider the relation between mitochondrial and nuclear DNA and the genetic function of cytoplasmic DNA in general. Bollum rather briefly discusses the replication of DNA while Martin attempts to reconcile the large volume of work on the replication of viral RNA in various systems. The genetic code and mechanisms of protein synthesis are considered in four papers given by Khorana, by Matthaei and his colleagues, by Ochoa and his collaborators, and by Monro, Maden and Traut. Littauer and Revel describe their work on the possible function of the methyl groups in transfer RNA. The only genetic map in the book is given by Yaniv and Gros in their fascinating paper on modified aminoacyl RNA synthetases in temperature conditional mutants of Escherichia coli. The discussion which followed each paper is recorded and this includes some pertinent invited comments by Leder and Clark on synonymous codewords and on phasing mechanisms.

The book has been produced well, although the print is more difficult to read than the styles of type that are usually used by Academic Press. Although the numbering of pyrimidine rings is only referred to occasionally, it is unfortunate that among the various papers two different numbering conventions have been used.
As a whole, the book gives an impression of high scientific quality. New information is presented and discussed in a stimulating and balanced manner in relation to pertinent work from other laboratories. Many useful references are given and there is both a subject and an author index. It should prove to be a valuable guide to many aspects of nucleic acid biochemistry.

K. Burton

\section{INVERTEBRATE NEUROPHYSIOLOGY}

Invertebrate Nervous Systems: their Significance for Mammalian Neurophysiology

Edited by C. A. G. Wiersma. Pp. ix +370 . (Chicago and London: The University of Chicago Press, 1967.) $\$ 10 ; 728$. net.

THIs symposium volume contains twenty-seven papers on various aspects of invertebrate neurophysiology. About half of these, comprising just over two-thirds of the book, review recent progress in limited parts of the field. This type of article seems to be particularly suited to inclusion in a symposium volume, because it is less extensive and more detailed than the orthodox review article. The remainder of the book consists of brief reports on particular investigations, the matter of which, I suspect, will shortly appear at greater length in their proper place, in journals devoted to the publication of original observations.

The longer articles between them cover a considerable slice of invertebrate neurophysiology. G. A. Kerkut gives a useful account of recent work on the pharmacology and ionic bases of synaptic transmission in molluscan neurones, and F. Strumwasser considers the types of information that may be stored in them. M. J. Cohen describes his work on the RNA metabolism of regenerating insect neurones. Some of the ways in which the nervous system controls the development of arthropods are described by J. S. Edwards. I. R. Hagadorn gives a brief review of neurosecretory mechanisms, and I. M. Cooke describes his experiments on the relation between electrical activity and secretion in neurosecretory axons. Nonstriated muscles are examined by C. L. Prosser, and G. Hoyle delivers a polemic against the assumption that all striated muscles are like the frog sartorius.

All these different approaches are concerned, more or less, with physiological analysis at the cellular level. The usefulness of invertebrate material for this type of study is two-fold. First, many individual invertebrate neurones are larger and more accessible than those of vertebrates. Consequently, certain physiological problems may be much more easily solved by the use of invertebrate material; our knowledge of the mechanism of nervous conduction, to pick the most obvious example, would be much less advanced than it is if it were not for the giant axons of squids. Second, the study of a variety of different types of cells serves to indicate the variety of possible types of neuronal activity. An example of this is provided by the discovery of electrically transmitting synapses in invertebrates; the possibility of such electrical transmission must now be borne in mind by anyone investigating neuronal interaction in vertebrates.

Other approaches are concerned with the properties of systems rather than individual units. Here the great advantage of most invertebrate nervous systems is their relative simplicity in comparison with those of vertebrates. Their investigation, therefore, may provide us with models of how parts of the vertebrate nervous system work. The remainder of this volume is concerned with studies of this type.

G. M. Hughes and W. D. Chapple describe the interconnexions of neurones in the ventral nerve cords of crustaceans and the ganglia of gastropod molluses. The organization of central ganglia is discussed by D. M. Maynard in an article which includes a most interesting comparison of the control of cephalopod colour patterns 